\title{
Editors' Comment
}

\section{Kiyoshige Ohseto and Hiroyuki Uchino}

\subsection{Neck Comment}

The landmark (blind) method for brachial plexus block is currently not recommended in pain clinics because of its rather frequent association with complications. However, the landmark method is helpful for identifying the optimum site for needle insertion, as viewed from the skin surface, and for determining the needle tip direction. The ultrasound-guided brachial plexus block is easier for beginners than the landmark technique if they marked the ideal needle insertion point on the skin surface in advance.

The ultrasound-guided method provides visualization of the nerve and vessels allowing local anesthetic injection into the brachial plexus; in addition, brachial plexus block can be simply performed at the bedside. On the other hand, the disadvantages of this technique include inability to visualize local anesthetic injection into small vessels and to clearly show the extent of local anesthetic spread in a craniocaudal direction.
In contrast, fluoroscopy-guided brachial plexus block uses local anesthetic mixed with contrast media, allowing real-time confirmation of contrast media spreading and of anesthetic injection into the vessels. This fluoroscopy-guided method allows injection of a high concentration and a large volume of local anesthetics during surgery and in patients with severe pain. The disadvantage of this technique is X-ray exposure.

\subsection{Neck Comment in Acraniocaudal Direction}

When treating the neck, the ultrasound-guided method allows the performance of nerve blocks. Using this method, a majority of nerve blocks can be more safely performed in a shorter time than using previously used landmark- and fluoroscopyguided methods. For brachial plexus block, the ultrasoundguided method particularly allows physicians to inject drug solutions directly into the painful nerves at the bedside.

K. Ohseto $(\bowtie) \cdot$ H. Uchino

Department of Anesthesiology, Tokyo Medical University,

Tokyo, Japan

e-mail: ohseto@tokyo-med.ac.jp 